\title{
Universiteit
}

Leiden

The Netherlands

\section{Collaborative mental health care versus care as usual in a primary care setting: A randomized controlled trial.}

Orden, M. van; Hoffman, T.; Haffmans, P.M.J.; Spinhoven, P.; Hoencamp, E.

\section{Citation}

Orden, M. van, Hoffman, T., Haffmans, P. M. J., Spinhoven, P., \& Hoencamp, E. (2009).

Collaborative mental health care versus care as usual in a primary care setting: A randomized controlled trial. Psychiatric Services, 60, 74-79. Retrieved from https://hdl.handle.net/1887/15701

Version: $\quad$ Not Applicable (or Unknown)

License: $\quad$ Leiden University Non-exclusive license

Downloaded from: https://hdl.handle.net/1887/15701

Note: To cite this publication please use the final published version (if applicable). 


\author{
Mirjam van Orden, M.Sc. \\ Tonko Hoffman, M.Sc. \\ Judith Haffmans, Ph.D. \\ Philip Spinhoven, Ph.D. \\ Erik Hoencamp, Ph.D., M.D.
}

Objective: This study compared the effectiveness of treating common mental disorders in a collaborative care program in a primary care setting and the effectiveness of treating such disorders through traditional referral of patients to mental health services. Methods: In a cluster randomized controlled trial, 27 general practitioner practices in the Netherlands were designated to provide either collaborative care or usual care. In the collaborative care condition, a mental health care professional worked on site at the primary care practice and was available to provide patients a maximum of five appointments if they were referred by the general practitioner. If indicated, referral to specialized mental health services followed. In the usual care condition, if indicated, general practitioners would refer patients to off-site specialized mental health services. The study included 165 patients. At baseline and at three, six, and 12 months, the study assessed patients' psychopathology, patients' quality of life, and patients' and general practitioners' satisfaction with the treatment provided. Delay in seeing a mental health provider, duration of treatment, number of appointments, and related treatment costs were assessed at 12 months. The data were analyzed with hierarchical linear models. Results: Level of patients' psychopathology and quality of life significantly improved over time, and there were no significant differences between models of care. There was no significant difference in patients' satisfaction with care in either condition. The collaborative care condition resulted in significantly higher satisfaction with services among general practitioners, shorter referral delay, reduced time in treatment, fewer appointments, and consequently lower treatment costs. Conclusions: Collaborative care for a heterogeneous group of persons with common mental disorders seems to be as effective as the usual practice of referral to mental health services for reducing psychopathology, but it is significantly more efficient regarding referral delay, duration of treatment, number of appointments, and related treatment costs. (Psychiatric Services 60:74-79, 2009)

Ms. van Orden, Mr. Hoffman, Dr. Haffmans, and Dr. Hoencamp are affiliated with the Research Department, Parnassia Bavo Group, Monsterseweg 83, the Hague, 2553 RJ, Netherlands (e-mail: m.vanorden@parnassiabavogroep.nl).Dr. Haffmans and Dr. Hoencamp are also with the Department of Psychology, Leiden University, Leiden, Netherlands, with which Dr. Spinhoven is affiliated.
I $\mathrm{n}$ the Netherlands, the general practitioner acts both as a mental health care provider and as a gatekeeper. As a gatekeeper, the general practitioner can refer patients for mental health care that is more specialized. Over the past 20 years there has been an increase in general practitioner referrals to mental health services. In response the Dutch government has strengthened the general practitioner's gatekeeper function to manage and limit the ever-increasing number of people using mental health care services (1). The government did this by specifying in the insurance guidelines that a specialist in nonacute care can start treatment only after receiving a formal written referral by a general practitioner. Yet general practitioners, already struggling with increasing workloads, needs of patients, and long working hours (2), have tended increasingly to refer patients with mental health problems to mental health services (3). This has resulted in long waiting times, poor continuity of care, and increasing dissatisfaction among patients, general practitioners, and mental health professionals (4). To decrease both the workload of the general practitioner and referrals to specialized mental health services, various forms of collaborative mental health care have been introduced (2).

Three collaborative care models have been described: the shifted outpatient clinic, the consultation liaison model, and the attached-mental 
health professional model (liaison attachment scheme) $(5,6)$. In the third model, mental health professionals are attached to a primary care practice and operate as part of the extended primary care team, leading to co-located services. This model results in improved geographical convenience for patients, decreased stigma, increased ease of referral, increased communication, and better continuity and integrated care $(5,6)$.

The literature concerning the feasibility and effectiveness of the various collaborative care models mainly addresses specific psychiatric conditions. Collaborative care interventions have proved to be effective in reducing psychopathology among persons with depression $(7,8)$, including elderly persons (9). Studies involving patients with psychosis, patients who abuse substances, and patients who use a high volume of mental health care have yielded mixed results. Studies of patients with anxiety disorders, personality disorders, eating disorders, attention-deficit disorder, and dementia are underrepresented (10). Collaborative care studies of heterogeneous patient groups with common mental disorders are scarce. Only two randomized controlled trials, both carried out in the United Kingdom, have been conducted $(11,12)$. The studies found a significant improvement in mean patient symptom scores, social functioning, and quality of life, with no significant differences between the usual and collaborative care conditions.

This study compared the effect of introducing collaborative care based on the attached-mental health professional model in a primary care setting in the Netherlands. We hypothesized that collaborative care would be at least equal to usual care regarding the effect on the psychopathology of patients, quality of life of patients, and satisfaction of patients and general practitioners, but it would be superior with regard to referral delay, duration of treatment, number of appointments, and related treatment costs.

\section{Methods}

Approval for the study was obtained from an Independent National Review Board for Mental Health (METIGG) in Utrecht.

\section{Randomization}

We invited all general practitioner practices in the Hague metropolitan area (126 practices with 240 general practitioners) that were not already participating in an ongoing collaborative care program to participate in this study. Of the 42 eligible practices, 15 declined to participate, and the remaining 27 practices (with 46 general practitioners) were randomly assigned to groups-14 practices were assigned to the collaborative care condition and 13 were assigned to care as usual. [A diagram showing the participating practices and number of patients referred to the trial is available as an online supplement at ps. psychiatryonline.org.] In this cluster randomized controlled trial, practices were the unit of randomization $(13,14)$. The practices (not individual patients) were randomly assigned to one of two conditions: either the practice continued its usual way of referring patients to specialized mental health services if indicated (usual care), or the practice referred patients to an on-site mental health professional who could see the patient for a maximum of five sessions (collaborative care). Between January 2003 and March 2005 all participating practices reported to the researchers all patients whom they intended to refer to a mental health organization.

\section{Study population}

Inclusion criteria for patients were age 18 or older, the presence of a mental disorder, and an indication for treatment that is more specialized. Exclusion criteria were dementia, delirium, acute severe psychotic symptoms, or a crisis condition demanding immediate care. The enrolled patients $(\mathrm{N}=165)$ reflect an urban population, and all were insured under the near-universal Dutch health insurance system. Of the 165 patients, 133 (81\%) were native Dutch, ten $(6 \%)$ were from Surinam, four $(2 \%)$ were Turkish, three $(2 \%)$ were Moroccan, and 15 (9\%) were born in various other countries. According to the Mini International Neuropsychiatric Interview (MINI) $(15,16)$, the study group consisted of a heterogeneous group with common mental disorders, mainly mood and anxiety disorders (Table 1).
After oral and written information was presented to the patient, written informed consent was obtained. Neither patients nor general practitioners received financial or other incentives to participate.

\section{Assessments}

In this study the official Dutch version of the MINI 5.0.0. was used (17). Patients were interviewed by an independent, well-trained research nurse. The Symptom Checklist (SCL-90) (18) is a validated self-report inventory with 90 items measuring psychopathology during the previous week. The overall psychoneuroticism score was used (19). The World Health Organization Quality of Life Questionnaire (WHOQOL-BREF) (20) is a validated scale and produces scores in four domains related to quality of life and one domain referred to as the general evaluative facet, which describes overall quality of life and general health. The Dutch Mental Healthcare's "Thermometer of Satisfaction" is a widely used 20-item questionnaire that assesses patient satisfaction with mental health care received (21). The general practitioner completed a Likert scale survey with four items indicating satisfaction with regard to time saving, workload relief, change in patients' complaints, and change in patients' quality of life.

At baseline, the MINI, SCL-90, and WHOQOL-BREF were administered. At three, six, and 12 months the SCL-90, WHOQOL-BREF, and patient and general practitioner satisfaction surveys were given. Waiting time between referral and the first face-toface contact with a mental health professional (referral delay), duration of the treatment, and the number of mental health care appointments and their costs in Euros were assessed by telephone interviews with patients, by the electronic patient record system of the mental health services in the Hague, and by the rates provided by the Dutch Health Care Authority.

\section{Interventions}

In the collaborative care program, trained mental health professionals (community psychiatric nurses and psychologists) from mental health services had regular face-to-face con- 
Table 1

Sociodemographic characteristics of patients with mental disorders who were seen by general practitioner practices in the Netherlands, by type of care provided

\begin{tabular}{|c|c|c|c|c|c|c|}
\hline \multirow[b]{3}{*}{ Characteristic } & \multirow{2}{*}{\multicolumn{2}{|c|}{$\begin{array}{l}\text { Study } \\
\text { nonparticipants } \\
(\mathrm{N}=71)\end{array}$}} & \multicolumn{4}{|c|}{ Study participants ${ }^{\mathrm{a}}$} \\
\hline & & & \multicolumn{2}{|c|}{$\begin{array}{l}\text { Collaborative care } \\
(\mathrm{N}=102)\end{array}$} & \multicolumn{2}{|l|}{$\begin{array}{l}\text { Usual care } \\
(\mathrm{N}=63)\end{array}$} \\
\hline & $\mathrm{N}$ & $\%$ & $\mathrm{~N}$ & $\%$ & $\mathrm{~N}$ & $\%$ \\
\hline Age $\left(\mathrm{M}_{ \pm} \mathrm{SD}\right)^{\mathrm{b}}$ & \multicolumn{2}{|l|}{$36.1 \pm 15.0$} & \multirow{2}{*}{\multicolumn{2}{|c|}{$40.2 \pm 14.3$}} & \multirow{2}{*}{\multicolumn{2}{|c|}{$40.4 \pm 12.9$}} \\
\hline Gender & & & & & & \\
\hline Male & 25 & 35 & 29 & 28 & 24 & 38 \\
\hline Female & 46 & 65 & 73 & 72 & 39 & 62 \\
\hline \multicolumn{7}{|l|}{$\begin{array}{l}\text { Duration of complaints } \\
\text { before referral }^{c}\end{array}$} \\
\hline$<3$ months & 19 & 30 & 23 & 23 & 18 & 31 \\
\hline 3-9 months & 17 & 27 & 30 & 30 & 13 & 22 \\
\hline >9 months & 27 & 43 & 47 & 47 & 27 & 47 \\
\hline \multicolumn{7}{|l|}{ Mini International } \\
\hline \multicolumn{7}{|l|}{ Neuropsychiatric } \\
\hline \multicolumn{7}{|l|}{ Interview diagnosis } \\
\hline Mood disorder & & & 33 & 32 & 19 & 30 \\
\hline Anxiety disorder & & & 33 & 32 & 28 & 44 \\
\hline Addiction & & & 1 & 1 & 2 & 3 \\
\hline Eating disorder & & & 2 & 2 & 1 & 2 \\
\hline Psychotic disorder & & & 1 & 1 & 2 & 3 \\
\hline Suicidal ideation & & & 7 & 7 & 2 & 3 \\
\hline Adjustment disorder & & & 25 & 25 & 9 & 14 \\
\hline
\end{tabular}

${ }^{a}$ No significant differences were found between care conditions.

b Significant difference between nonparticipants and participants $(\mathrm{t}=2.09, \mathrm{df}=233, \mathrm{p}=.037)$.

${ }^{c}$ Not all data were available for all persons.

tact with the general practitioner, consulted patients, and helped to refer patients in need of acute care. In the general practitioner's office, patients who were referred to the attached mental health professional received a short focused intervention consisting of a clinical assessment. This was followed by a maximum of four sessions based on cognitive-behavioral therapy and supporting principles (22). A team of psychiatrists met face to face with the mental health professionals once a month and conducted regular meetings with the general practitioners. If indicated, patients could be referred to a specialized mental health care program after the initial clinical assessment with the mental health professional or at a later date.

Usual care involved the traditional referral of patients by the general practitioner, if indicated, to mental health services for treatment.

\section{Analysis}

Outcome measures included patients' psychopathology, patients' quality of life, patients' and general practitioners' satisfaction with care, system-related waiting time before treatment (referral delay), duration of the treatment, and the number of mental health care appointments and their costs in the 12-month follow-up after referral. For a study where the patients were randomly assigned to treatment, the Power and Precision software (Biostat) determined that 82 patients per condition should be needed to detect a clinically relevant difference in mean $\pm \mathrm{SD}$ scores between the two conditions of $22 \pm 50$ points on the SCL-90 (power $80 \%$, $\alpha=.05)$. Taking into account an intracluster correlation coefficient (ICC) of .01 for the cluster randomization (23), the needed sample size per condition was 85 to 89 patients.

Preliminary analyses included checking for selection bias and the computation of descriptive statistics, chi square analyses, and t tests to assess the comparability of study groups at baseline. Five patients (two in collaborative care, three in usual care) had no contact with any mental health service during the 12 months after referral by their general practitioner. These patients were included in the analyses (intention-to-treat principle).

A consequence of cluster randomization at the level of general practitioner practices (instead of at the level of individual patients) was a lack of independence for the outcomes of patients from the same practice. This means that outcomes for patients from the same practice were correlated-that is, nonindependent (13). Ignoring clustering and dependence of outcome could create serious technical problems-for example, underestimation of standard errors and regression coefficients (24). Therefore hierarchical linear modeling was chosen as the statistical method to resolve these issues (24). Analyses were carried out with MlwiN 2.0 (25). A hierarchical structure with three levels was identified in the data: each patient had several repeated measurements of outcomes, and several patients were referred by the same general practitioner. The repeated measurements per patient were assigned to level 1 , the patients to level 2 , and the general practitioners to level 3. Units at one level were grouped, or nested, within units at the next highest level.

In the analyses four dependent variables were used: the SCL-90 sum score as an indicator of overall psychopathology, WHOQOL-BREF general evaluative facet regarding quality of life, patients' overall satisfaction rating, and the mean score on the four items indicating general practitioners' satisfaction. These variables were analyzed separately; the satisfaction of general practitioners and patients was assessed at three time points, whereas psychopathology and quality of life were assessed at four time points. To examine the effects of the variables, a conceptual model for a general time effect was constructed. The relative effects of condition and its time effect were examined by entering them in the basic model. Variables resulting in a significant improvement of the conceptual model were retained. See Raudenbush and Bryk (24) for a formal description of the analysis, or contact the authors of this article for more information. 
Outcomes of patients with mental disorders who were seen by general practitioner practices in the Netherlands, by type of care provided

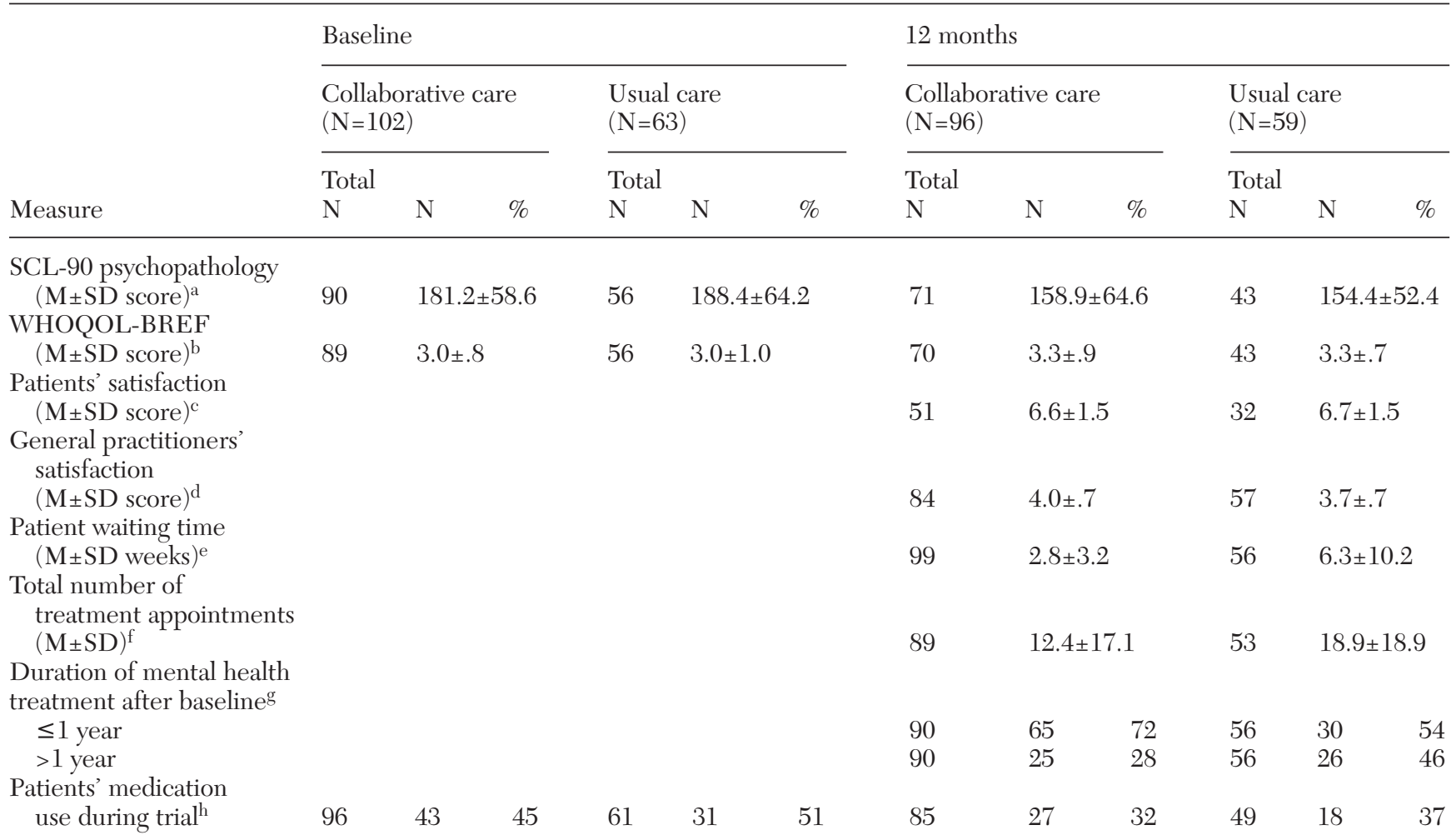

a As measured by the psychopathology subscale on the 90-item Symptom Checklist. Possible scores range from 90 to 450 , with higher scores indicating more psychological distress. No difference between groups at baseline.

${ }^{b}$ As measured by the general evaluative facet domain on the World Health Organization Quality of Life Questionnaire. Possible scores range from 1 to 5 , with higher scores indicating a higher perceived quality of life. Collaborative care group compared with usual care group at baseline: $\mathrm{t}=.426, \mathrm{df}=143, \mathrm{p}=.671$

${ }^{c}$ As measured by the patients' overall satisfaction rating on the Dutch Mental Healthcare's Thermometer of Satisfaction. Possible scores range from 1 to 10 , with higher scores indicating higher satisfaction with care.

d As measured by the mean score on the four items on the general practitioners' satisfaction survey. Possible scores range from 1 to 5 , with higher scores indicating higher satisfaction with care of the referred individual patients.

e $\mathrm{t}=-2.5, \mathrm{df}=61, \mathrm{p}=.016$

${ }^{\mathrm{f}} \mathrm{t}=-2.1, \mathrm{df}=140, \mathrm{p}=.04$

g $\chi^{2}=5.3, \mathrm{df}=1, \mathrm{p}=.02$

$\mathrm{h}$ No difference between groups

\section{Results}

The 27 participating general practitioner practices used standard forms to identify for the researchers $236 \mathrm{pa}-$ tients who were eligible for inclusion in the study. Of these patients 165 consented to participate: 102 of the patients were being seen at practices in the collaborative care group, and 63 were from practices in the usual care group.

The imbalance between the two conditions with regard to the number of patients referred to mental health services is noteworthy. A difference in the number of referrals between the general practitioners practices was already apparent two years before the start of the study and persisted two years after the start of the trial. This consistency indicates that the difference is a reflection of a historical referral pattern and is not related to this study.

The 165 participating patients did not differ from the 71 patients who declined to participate in the study except in regard to age: participants were significantly older $(40.3 \pm 13.7$ versus $36.1 \pm 15.0$ years). There were no differences between the usual care group and the collaborative care group on any of the other baseline variables (Tables 1 and 2 ).

As shown in Table 2, the number of patients using medication (antidepressants, benzodiazepines, analgesics, or antipsychotics) did not differ between conditions (collaborative care or care as usual) at baseline or at the end of the trial. The mean \pm SD waiting time for the first face-to-face contact with a mental health professional was significantly lower for collaborative care $(2.8 \pm 3.2$ weeks $)$ than for usual care $(6.3 \pm 10.2$ weeks $)$, according to a t test for independent samples. In the collaborative care condition, 33 patients $(32 \%)$ were subsequently referred to specialized mental health care after one or more appointments with the mental health professional. For the patients who were subsequently referred, the mean number of appointments with the attached-mental health professional in the collaborative care group was $3.5 \pm 4.2$. The patients who were subsequently referred to specialized mental health care by the collabora- 
Table 3

Multilevel estimates of primary outcome measures among patients with mental disorders who were seen by general practitioner practices in the Netherlands

\begin{tabular}{|c|c|c|c|}
\hline Variable $^{a}$ & Estimate & SE & $\mathrm{p}$ \\
\hline \multicolumn{4}{|l|}{ SCL-90 psychopathology sum score } \\
\hline Average initial psychopathology (intercept) & 167.624 & 7.805 & .001 \\
\hline Average improvement rate & -2.210 & .417 & .001 \\
\hline Average condition effect & -1.181 & 14.333 & .934 \\
\hline $\begin{array}{l}\text { Interaction of improvement rate } \\
\text { and condition effect }\end{array}$ & .868 & .772 & .263 \\
\hline \multicolumn{4}{|l|}{ WHOQOL-BREF } \\
\hline Average initial quality of life (intercept) & 3.217 & .096 & .001 \\
\hline Average improvement rate & .027 & .006 & .001 \\
\hline Average condition effect & -.069 & .177 & .697 \\
\hline $\begin{array}{l}\text { Interaction of improvement rate and } \\
\text { condition effect }\end{array}$ & -.016 & .011 & .148 \\
\hline \multicolumn{4}{|l|}{ Patients'satisfaction } \\
\hline Average initial satisfaction (intercept) & 6.521 & .165 & .001 \\
\hline Average improvement rate & .007 & .023 & .761 \\
\hline Average condition effect & .195 & .303 & .521 \\
\hline $\begin{array}{l}\text { Interaction of improvement rate and } \\
\text { condition effect }\end{array}$ & -.023 & .042 & .585 \\
\hline \multicolumn{4}{|l|}{ General practitioners' satisfaction } \\
\hline Average initial satisfaction (intercept) & 3.838 & .062 & .001 \\
\hline Average improvement rate & .000 & .009 & 1.000 \\
\hline Average condition effect & .436 & .114 & .001 \\
\hline $\begin{array}{l}\text { Interaction of improvement rate and } \\
\text { condition effect }\end{array}$ & -.011 & .016 & .493 \\
\hline
\end{tabular}

a SCL-90, 90-item Symptom Checklist; WHOQOL-BREF, World Health Organization Quality of Life Questionnaire, general evaluative facet

tive care professional had an additional mean number of $21.7 \pm 24.3$ appointments.

According to a t test for independent samples, the total mean number of appointments in the 12-month period was significantly lower in the collaborative care condition $(12.4 \pm 17.1)$ than in the usual care condition $(18.9 \pm 18.9)$. The mean cost for a patient in the collaborative care condition was $€ 1,199 \pm 1,621$, and it was $€ 1,762 \pm 1,683$ in the usual care condition. At the end of the 12-month period, significantly more participants from the collaborative care condition ( $\mathrm{N}=65$, or $72 \%$ ) were no longer participating in treatment, compared with participants from the usual care condition ( $\mathrm{N}=30$, or $54 \%)$, according to a chi square test (Table 2).

As shown in Table 3, a significant improvement over time was found for both the SCL-90 psychopathology score $(\mathrm{p}=.001)$ and the WHOQOLBREF general evaluative facet $(p=$ .001). However, there was no significant difference between the two conditions, and there was no interaction effect between improvement and condition. Patient satisfaction showed no significant improvement over time, no condition effect, and no interaction effect between improvement and condition. General practitioners' satisfaction did not improve significantly over time, and there was no interaction effect between improvement and condition. However, a significant difference was found between the conditions. General practitioners from the collaborative care condition had a satisfaction score that was significantly higher than that of general practitioners from the usual care condition ( $\mathrm{p}=.001)$ (Table 3$)$.

\section{Discussion}

We expected collaborative care to be equally or more effective than usual care in reducing psychopathology and increasing quality of life, patients' satisfaction, and general practitioners' satisfaction. We found that both collaborative care and usual care significantly reduced existing psychopathology, increased quality of life, and resulted in comparable satisfaction of patients. Collaborative care led to significantly higher levels of general practitioners' satisfaction and a significant reduction in referral delay and duration of treatment. A significant reduction of contacts with mental health organizations was achieved in the 12-month period, leading to a significant reduction in costs.

Our results are in accordance with the two randomized controlled trials comparing generic community mental health nursing care and problemsolving treatment with usual primary care $(11,12)$. Problem-solving treatment is a brief (six session) structured psychological treatment that has been developed. In another pilot study, enhanced liaison between secondary mental health care and primary care teams did not lead to a significant difference in the reduction of symptoms, compared with standard care (26). Although our study was slightly underpowered, the comparable effectiveness of both service models in decreasing patients' symptoms and increasing their quality of life may be a reflection of the high standard of primary care in the Netherlands. Consequently, it might be hard for an intervention to achieve significantly higher effectiveness than usual care (1). Additionally, the fact that the care in the collaborative care condition was provided by mental health professionals trained and supervised within the context of a mental health organization might account for the comparability of outcome in both conditions. Of course it might also be a reflection of the natural course of the disorders, in which symptoms may improve over time.

There are limitations to this study. When the study started in 2003, about $65 \%$ of the general practitioners in the Hague area were already participating in the collaborative care program. The remaining general practitioners who wanted to participate first had to enroll in this study. This may have resulted in a bias, because being assigned to the usual care condition might have created dissatisfaction for the general practitioner involved. Also, the number of referrals per general practitioner practice in the usual care condition was substantially less than that 
for the collaborative care condition. However, when this number was compared with the referral pattern two years before starting and two years after the trial, there was no change in the number of patients referred. In hindsight, stratification by number of earlier referrals at the start of the study could have prevented the risk of a possible preexisting referral bias. The fact that patients from both conditions were comparable on all baseline variables indicates that the randomization at the level of the general practitioner practice resulted in a successful randomization at the individual patient level. Finally, this study cannot answer whether other collaborative care programs might be more or less cost-effective than this particular collaborative intervention.

A strength of this study is that randomization occurred at the general practitioner level. This design permits comparison of general practitioner practice referrals, rather than the individual course of treatment per patient. The high follow-up rate and low drop-out rate are indications of the quality of the trial management. Because patients in the study had various common mental disorders - the types of disorders that are regularly seen by general practitioners - the findings of our study are generalizable to other general practitioners.

This study is the first randomized controlled trial comparing collaborative care and usual care among patients in an urban environment. Results might be generalized to other urban settings and countries with similar health care systems, such as the United Kingdom, Spain (27), and Canada (28).

\section{Conclusions}

This trial provides evidence that collaborative care for patients with common mental disorders at the primary care level is feasible and as effective as standard mental health care. However, collaborative care results in significantly lower utilization of resources, as indicated by fewer appointments with a mental health professional, shorter treatment duration, and higher satisfaction among general practitioners.

\section{Acknowledgments and disclosures}

This study was funded by Delta Lloyd and OHRA Ziekenfonds, a health insurance company. The authors thank the patients and general practitioners, interviewer L. Dijkhuizen, M.Sc., the staff and management of the collaborative care program, and the Parnassia Bavo Group.

The authors report no competing interests.

\section{References}

1. Schene AH, Faber AM: Mental health care reform in the Netherlands. Acta Psychiatrica Scandinavica: Supplementum (410):74-81, 2001

2. Verhaak PF, Hoeymans N, Garssen AA, et al: Mental health in the Dutch population and in general practice: 1987-2001. British Journal of General Practice 55:770-775, 2005

3. Verhaak PF, van de Lisdonk EH, Bor JH, et al: GPs' referral to mental health care during the past 25 years. British Journal of General Practice 50:307-308, 2000

4. De Vries W: Consultation in primary care: know-it-all or gift from heaven? [in Dutch]. Huisarts en Wetenschap 44:559-562, 2001

5. Craven MA, Bland R: Shared mental health care: a bibliography and overview. Canadian Journal of Psychiatry 47:iS-viiiS, 1S-103S, 2002

6. Gask L, Sibbald B, Creed F: Evaluating models of working at the interface between mental health services and primary care British Journal of Psychiatry 170:6-11, 1997

7. Bower P, Gilbody S, Richards D, et al: Collaborative care for depression in primary care: making sense of a complex intervention: systematic review and meta-regression British Journal of Psychiatry 189:484-493, 2006

8. Katon W, Von Korff M, Lin E, et al: Collaborative management to achieve treatment guidelines: impact on depression in primary care. JAMA 273:1026-1031, 1995

9. Unützer J, Katon W, Callahan CM, et al: Collaborative care management of late-life depression in the primary care setting: a randomized controlled trial. JAMA 288:28362845,2002

10. Craven MA, Bland R: Better practices in collaborative mental health care: an analysis of the evidence base. Canadian Journal of Psychiatry 51:7S-72S, 2006

11. Gournay K, Brooking J: Community psychiatric nurses in primary health care. British Journal of Psychiatry 165:231-238, 1994

12. Kendrick T, Simons L, Mynors-Wallis L, et al: Cost-effectiveness of referral for generic care or problem-solving treatment from community mental health nurses, compared with usual general practitioner care for common mental disorders: randomised controlled trial. British Journal of Psychiatry 189: $50-59,2006$

13. Campbell MK, Grimshaw JM: Cluster randomised trials: time for improvement: the implications of adopting a cluster design are still largely being ignored. BMJ 317:11711172,1998
14. Campbell MK, Elbourne DR, Altman DG: CONSORT statement: extension to cluster randomised trials. BMJ 328:702-708, 2004

15. Sheehan D, Lecrubier Y, Sheehan K, et al: The Mini-International Neuropsychiatric Interview (MINI): the development and validation of a structured diagnostic psychiatric interview for DSM-IV and ICD-10. Journal of Clinical Psychiatry 59:22-33, 1998

16. Lecrubier Y, Sheehan D, Weiller E, et al: The Mini International Neuropsychiatric Interview (MINI): a short diagnostic structured interview: reliability and validity according to the CIDI. European Psychiatry 12:224-231, 1997

17. Overbeek T, Schruers K, Griez E: MINIThe International Neuropsychiatric Interview: Dutch version 5.0.0 DSM-IV. Maastricht, Netherlands, University of Maastricht, 1999

18. Derogatis LR, Lipman RS, Covi L: SCL-90: an outpatient psychiatric rating scale-preliminary report. Psychopharmacological Bulletin 9:13-28, 1973

19. Arrindell W, Ettema J: Manual of a Multidimensional Indicator of Psychopathology [in Dutch]. Lisse, Netherlands, Swets Test Publishers, 1986

20. Group TW: Development of the World Health Organization WHOQOL-BREF quality of life assessment. Psychological Medicine 28:551-558, 1998

21. Kok I, van Wijngaarden B: Client Appreciation in Mental Health Care: Manual of the Dutch Mental Healthcare Thermometer of Appreciation by Clients [in Dutch]. Utrecht, Trimbos-instituut/GGZ Nederland, 2003

22. Rijnders P, De Jong T, Isebaert L, et al: Short-Time Treatment in Mental Health Care: The Practice [in Dutch]. Houten/ Diegem, Bohn Stafleu Van Loghum, 2002

23. Killip S, Mahfoud Z, Pearce K: What is an intracluster correlation coefficient? Crucial concepts for primary care researchers. Annals of Family Medicine 2:204-208, 2004

24. Raudenbush S, Bryk A: Hierarchical Linear Models. Applications and Data Analysis Methods. Newbury Park, Calif, Sage, 2002

25. Rasbash J, Steele F, Browne W, et al: A User's Guide to MLwiN. Bristol, United Kingdom, University of Bristol, Centre for Multilevel Modeling, 2005

26. Emmanuel JS, McGee A, Ukoumunne OC, et al: A randomised controlled trial of enhanced key-worker liaison psychiatry in general practice. Social Psychiatry and Psychiatric Epidemiology 37:261-266, 2002

27. Verhaak PF, van den Brink-Muinen A, Bensing JM, et al: Demand and supply for psychological help in general practice in different European countries: access to primary mental health care in six European countries. European Journal of Public Health 14:134140,2004

28. Kates N, Craven MA, Crustolo AM, et al: Mental health services in the family physician's office: a Canadian experiment. Israelian Journal of Related Sciences 35:104 113,1998 